being explored for new approaches to reduction of plasma LDL cholesterol. Bile acids are synthesized from cholesterol in the liver and secreted into the intestine, whereupon they are reabsorbed and transported back to the liver (Fig. $1 b$ ). Clinical trials of inhibitors of the intestinal bile acid transporter (iBAT), which transports bile acids across the intestinal brush border and promotes their reabsorption, are in progress ${ }^{9}$. An analogous transporter of cholesterol and other sterols across the intestinal brush border is likely to exist (Fig. $1 b$ ) and a molecular inhibitor of intestinal cholesterol absorption reduces LDL cholesterol in animals and humans ${ }^{10}$. In addition, inhibition of the microsomal triglyceride transfer protein, which is required for the assembly and secretion of the nascent VLDL particle (Fig. $1 b$ ), results in markedly reduced plasma cholesterol in animals ${ }^{11}$. Therefore, several new approaches to re- duction of plasma LDL cholesterol are likely to be introduced over the next decade. Further elucidation of the molecular pathways that control sterol and lipid metabolism may yield additional targets for therapy, not only for the reduction of plasma LDL cholesterol, but also for disorders of fatty-acid, triglyceride and glucose metabolism as well.

1. Grundy, S.M. Statin trials and goals of cholesterollowering therapy. Circulation 97,1436-1439 (1998).

2. Executive Summary of The Third Report of The National Cholesterol Education Program (NCEP) Expert Panel on Detection, Evaluation, And Treatment of High Blood Cholesterol In Adults (Adult Treatment Panel III). JAMA 285, 2486-2497 (2001).

3. Grand-Perret, T. et al. SCAP ligands are potent new lipid lowering drugs. Nature Med. 7, 1332-1338 (2001).

4. Brown, M.S. \& Goldstein, J.L. A proteolytic pathway that controls the cholesterol content of membranes, cells, and blood. Proc. Natl. Acad. Sci. USA 96, 11041-11048 (1999).

5. Edwards, P.A., Tabor, D., Kast, H.R. \& Venkateswaran, A. Regulation of gene expression by SREBP and SCAP. Biochim. Biophys. Acta 1529,
$103-113(2000)$

6. Korn, B.S. et al. Blunted feedback suppression of SREBP processing by dietary cholesterol in transgenic mice expressing sterol-resistant SCAP(D443N). J. Clin. Invest. 102, 2050-2060 (1998).

7. Sheng, Z., Otani, H., Brown, M.S. \& Goldstein, J.L. Independent regulation of sterol regulatory elementbinding proteins 1 and 2 in hamster liver. Proc. Natl. Acad. Sci. USA 92, 935-938 (1995).

8. Repa, J.J. et al. Regulation of mouse sterol regulatory element-binding protein-1c gene (SREBP-1c) by oxysterol receptors, LXR $\alpha$ and LXR $\beta$. Genes Dev. 14, 2819-2830 (2000)

9. Izzat, N.N., Deshazer, M.E. \& Loose-Mitchell, D.S New molecular targets for cholesterol-lowering therapy. I. Pharmacol. Exp. Ther. 293, 315-320 (2000)

10. Bays, H.E. et al. Effectiveness and tolerability of ezetimibe in patients with primary hypercholesterolemia: pooled analysis of two phase II studies. Clin. Ther. 23, 1209-1230 (2001).

11. Wetterau, J.R. et al. An MTP inhibitor that normalizes atherogenic lipoprotein levels in WHHL rabbits. Science 282, 751-754 (1998).

Center for Experimental Therapeutics

University of Pennsylvania School of Medicine

Philadelphia, Pennsylvania, USA

Email:rader@mail.med.upenn.edu

\title{
Virus knocking at nucleus door
}

Viruses deliver their genomes to the host cell's nucleus where they can replicate. Like most viruses, the adenovirus 2 (Ad2) particle is far too large to squeeze through nuclear pores and the virus has had to find a clever way to 'squirt' its genome inside the nucleus. A study published in the latest issue of Nature Cell Biology reveals that $\mathrm{Ad} 2$ enlists the help of an unexpected cellular partner-histone $\mathrm{H} 1$ - to achieve its goal.

Urs Greber and colleagues at the University of Zürich show that $\mathrm{Ad} 2$ docks to the nuclear pore via the nuclear-pore complex (NPC) receptor
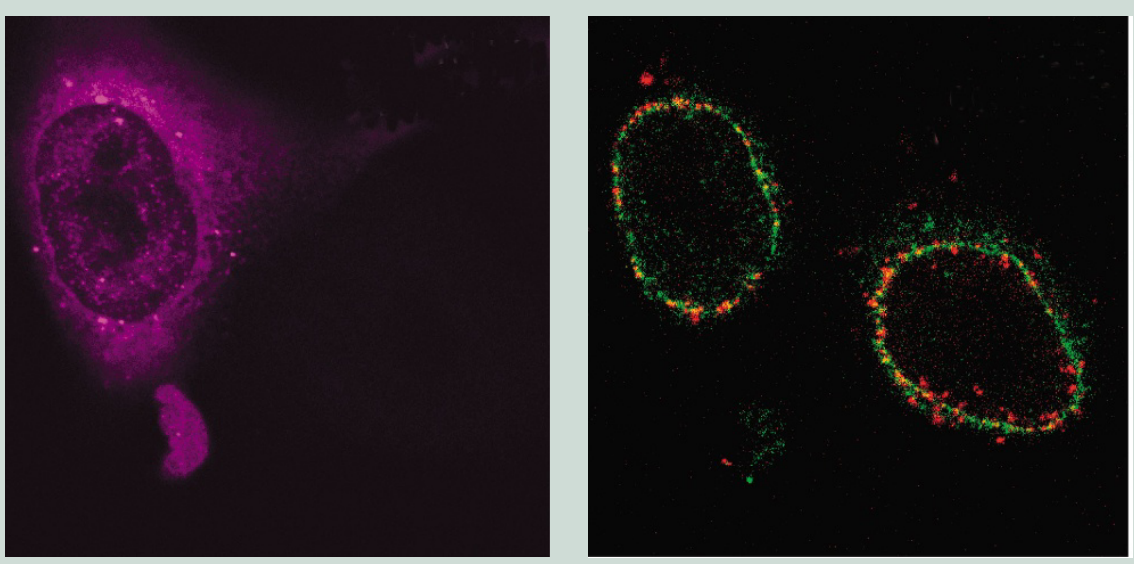
CAN/Nup214 - the first receptor of the nuclear membrane identified to bind any virus. What happens then is remarkable. The virus disassembles slowly and transfers its genetic material through the nuclear pore while remaining attached to the NPC. But the CAN/Nup214 receptor is not the only cellular protein required for virus disassembly; the virus also gets help from an unlikely ally - the linker histone $\mathrm{H} 1$ protein.

$\mathrm{H} 1$ is a generic chromatin-binding protein implicated in transcriptional repression and higher-order chromatin structure. $\mathrm{H} 1$ does not stably bind chromatin but rather bounces back and forth between chromatin and nucleoplasm, allowing a small, unbound fraction of $\mathrm{H} 1$ to escape from the nucleus. Under normal circumstances, the fugitive $\mathrm{H} 1$ is retrieved by the cell's import system and brought back into the nucleus; in Ad2-infected cells, it assists in Ad2 disassembly.

The photographs show two human lung carcinoma cells, one of which was injected with anti-histone $\mathrm{H} 1$ antibodies. The injected cell can be seen in the photograph on the left, stained with an injection marker present in the antibody solution (purple). The photograph on the right shows the two cells stained with antibodies to the NPC (green) and Ad2 DNA (red). In the uninjected cell, Ad2 DNA can be seen bound to NPC on the nuclear membrane but also inside the nucleus; in the cell injected with anti-H1 antibodies all Ad2 DNA remains bound to the NPC.

There is another twist to the story. $\mathrm{H} 1$ does not promote $\mathrm{Ad} 2$ disassembly by interacting with viral DNA, but rather with the hexon protein that is part of the viral capsid. Specifically, $\mathrm{H} 1$ most likely binds a surface-exposed acidic cluster on hexon, possibly destabilizing its interactions with other capsid components and triggering the viral disassembly process. In uninfected cells, $\mathrm{H} 1$ is efficiently retrieved from the cytoplasm by the $\mathrm{H} 1$ import factors importin $\beta$ and importin 7. As the disassembly of Ad2 also requires the same two importins, the authors speculate that hexon-bound $\mathrm{H} 1$ is recognized by the normal import system of the cell, and the complex is brought into the nucleus thereby destabilizing the viral capsid. The process triggers a gradual disassembly of the virus particle while it is held in place at the NPC, allowing the DNA that spills out of the virus to enter the nucleus.

LAURA BONETTA 